\title{
THE EXPERIENCE OF LIFESTYLE CHANGES AMONG MIDDLE EASTERN GRADUATE STUDENTS IN THE UNITED STATES
}

\author{
Salman Alzayani, $M D, M S c$ \\ Department of Family and Community Medicine \\ College of Medicine and Medical Sciences, Arabian Gulf University
}

\begin{abstract}
High rates of health problems have been reported among undergraduate students, with increased stress levels among college undergraduates in the United States in several fields of study. Academic life stresses are believed to play an important role in the health of students, which may affect their learning abilities and educational performance on top of their health
\end{abstract}

Aim Understanding the effect of cultural and environmental changes on individual's behavior.

Methods : Graduate students from the Middle East who spent two years in the United States were selected for interviews. Colaizzi's phenomenological descriptive method of inquiry was adopted.

Results: Thirty-eight statements were extracted from the interview and sorted into nine themes: Away from home: homesickness, school is challenging and stressful, it is not cold it is freezing, lack of physical activity, social norms vs. peer pressure, dietary patterns and available options, away from home and arriving to new home, time is money and day to day expenses. An exhaustive description was created from the themes and the formulated meanings and was adjusted and validated by the interviewees. The fundamental structure was developed accordingly.

Conclusion : Despite changes in culture and environment, students' behavior and lifestyle have been affected in different ways, some were positively affected like being more time efficient and focusing more on work, and some were negatively affected like being under stress, eating unhealthy diet and physical inactivity. Students were aware of the changes in their lifestyle and were always trying to modify them.

Keywords: Lifestyle, Students, United States, Middle East, Health 


\section{Introduction}

High rates of health problems have been reported among undergraduate students, with increased stress levels among college undergraduates in the United States in several fields of study. Academic life stresses are believed to play an important role in the health of students, which may affect their learning abilities and educational performance on top of their health (Al-Dabal, et al., 2010). This study is aimed to study the experience of lifestyle changes among Middle Eastern graduate students in the United States, using Colaizzi's phenomenological descriptive method of enquiry (Colaizzi, 1978), in order to explore the impact of lifestyle changes due to environmental and cultural change and how would that affect the behavior of the individual.

\section{Evolution of the study}

Students who pursue their degrees away from their cultural norms have different experiences than those who pursue their degrees at their home countries. This was explored in a study of international students in the United States, which raises some sensitive issues about the nature of cultural learning and the societal acceptance of diversity and differences. The researchers stated that the students' experiences appear to reflect the tensions that exist in the global context regarding such diversity and difference (Lee, 2004). Al-Dabal et al., (2010) concluded that " unhealthy lifestyles such as lack of exercise, insufficient sleep and poor self-health care among students could well have contributed to their worse physical status. Often the high pressure of studies and limited time to acquire vast knowledge prevent students from adopting a healthy lifestyle". Interestingly, it seems that "not the severity of negative life events but their cumulating determines health and behavioral negative effects" (Supranowicz, 2011).

Sedentary lifestyle and physical inactivity are pandemics and are leading causes of death in the world. (Kohl et al., 2012). A study concluded that weight gain does occur in the first year of university, but in this case the increase was small and transitory, while weight gain over the longer term is negatively related to the weight at which students begin university and predicted in part by eating behavior traits and physical activity levels (Finlayson, et al., 2012). Alzayani and Hamadeh (2011) have studied an aspect of medical students sedentary lifestyle which was the use of electronic entertainment devices like computers and mobile phones as part of a bigger study covering a wider range of lifestyle behaviors like dietary patterns, physical activity, sleeping pattern, tobacco smoking and alcohol consumption. The study showed that most medical students spent 1-4 hours daily working on their computers, using their mobile phones and watching TV and DVD. A smaller number of students performed these activities for 
less than one hour per day. One hundred and thirty (42.2\%) female students used their mobile phones for more than 2 hours per day, 95 (31.3\%) watched TV and DVD and 203 (66.1\%) used computers. This study was performed in Bahrain, a small country in the Middle East, where the students are traveling from nearby countries that share the same cultural values and environment, this study gives us an important indication of the effect of studying and accommodation environments on students lifestyle.

Deluca (2005) conducted a study on students from the Middle East, specifically from Jordan who are pursuing their degrees in the United States. The researcher concluded that some specific curricular and administrative interventions might have improved student adjustment and overall success. These included allowing an extended time for orientation to the social and academic environment and limiting course content in the first semester. Lee (2004) stated positively that these cultural differences have positive effects on students as well as direct international experience appears to offer ways of building student confidence, development and growth through the personal challenges involved. International experience offers a vital opportunity to explore other ways of thinking and living.

\section{Purpose}

I have connection to several students from the Middle East since their arrival day to the United States and I always keep observing them and how they adapt to the abrupt cultural changes. Throughout the two years prior to this study, I have observed tens of them, however, cultural shock was not an issue here. Instead there was a sense of instability and the detachment of the society at the early beginning mainly in lifestyle including dietary patterns, food selections, use of public transportation, sleep patterns and even weather differences. They usually adapt to these changes by the first year and become a way of life in the second. In this study, I would like to explore and understand their experience of lifestyle changes since they moved to the United States to pursue their graduate studies in New England, in order to develop proper programs and policies toward international students during their studies in the United States.

\section{Method}

\section{Descriptive phenomenology}

Colaizzi developed his descriptive phenomenology method (Colaizzi, 1978) "under the supervision of Giorgi, who produced a body of literature devoted to the ongoing articulation and demonstration of empirically based phenomenological research in psychology. Colaizzi's procedural modification of Giorgi's approach to enquiry involved a process of validating the findings with participants. This process required the researcher 
returning the analysis of transcripts to the respective participants for review. Additional information arising from clarification and/or elaboration by participants would necessitate inclusion in the final explication of findings. This procedural modification by Colaizzi of Giorgi's method expanded the process of phenomenological analysis contributing to advancing a rigorous approach to phenomenological enquiry" (Edward \& Welch, 2011) The method is illustrated in Figure 1.

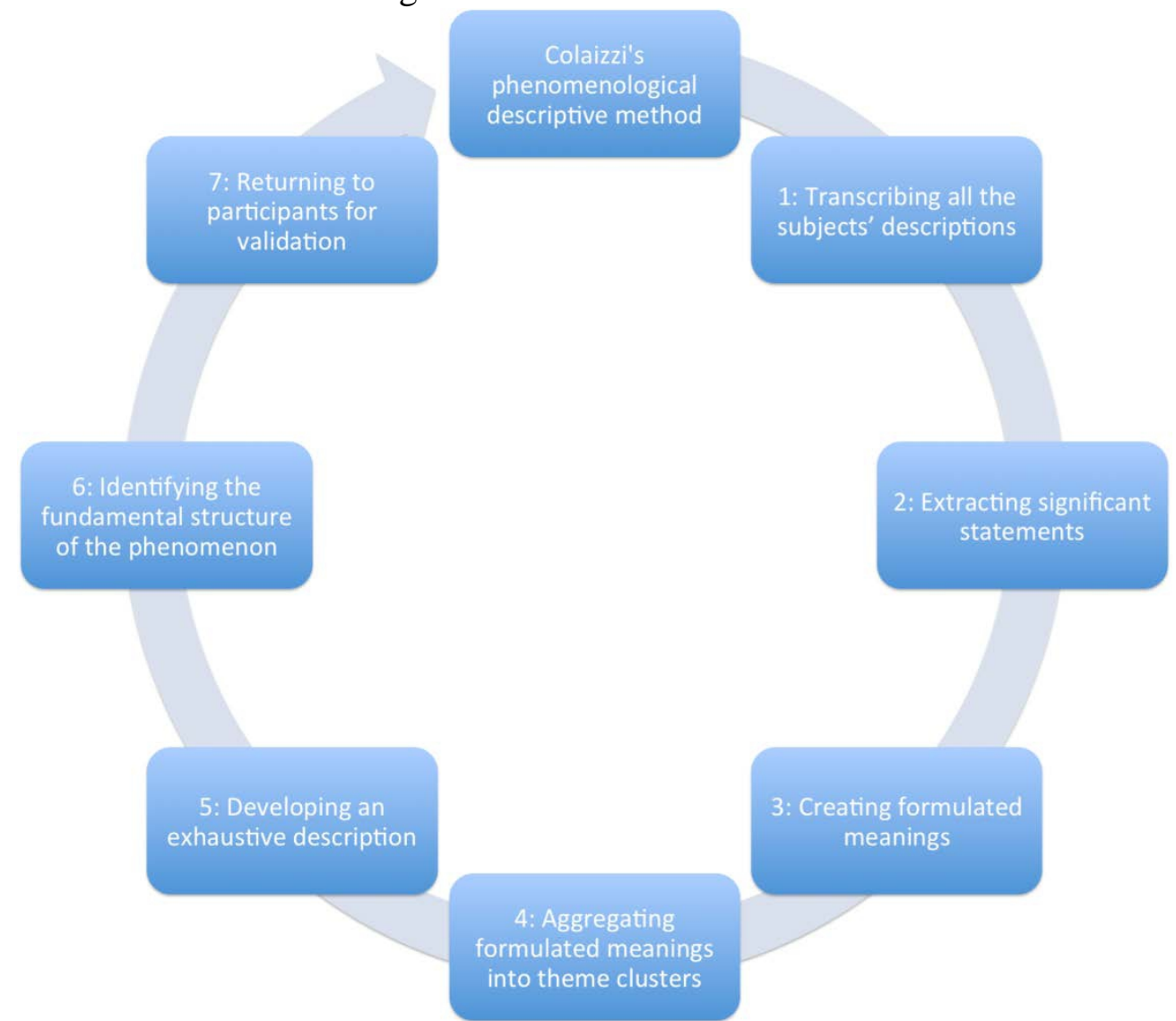

Figure 1. Procedures of Colaizzi’s phenomenological descriptive method (Colaizzi, 1978)

\section{Sample and setting}

Three graduate students from the Middle East who spent two years in the United States were selected for interviews, two males (25 and 29 year old) and one female (29 year old), all from Saudi Arabia. They were all enrolled as graduate students in the School of Engineering in a large university in New England. All subjects were married and living with their families in the United States during the time of the study. The subjects were recruited in the study by direct invitation by the researcher through social networks of the same university where the researcher belongs. Two 
interviews were conducted individually in a quiet room in one of the university building while one interview was conducted over the phone.

\section{General steps}

After contacting the proposed subjects, the researcher requested them to sign the consent form after explaining to them that their confidentiality and anonymity will be secured, and that they have the right to leave the study. After that the interviews were set individually in and according to the convenience of the subjects. Interviews were digitally taped after their permission. The subjects filled in demographic info sheets at the beginning of the interview. The opening statement of the interview was: "Please describe to me your lifestyle changes since you started your career as a graduate student in the United States. Share all your thoughts, feelings and perceptions you can recall until you have no more to say about the experience". This was the only question asked to the subjects as per the method guidelines used in this study (Colaizzi, 1978).

\section{Human subjects considerations}

The study was approved by the institutional review board for the university and students signed a consent form which has been approved by the IRB to participate in the study. They can end their participation at any time and the information they provided would be reported anonymously. The researcher has completed a required IRB course before conducting the study.

\section{Results}

Interviews transcription have been done by the researcher after completing all of the interviews which yielded 38 significant statements which have been extracted from the transcription and transferred into formulated meaning. Then these statements were sorted into 16 themes and then abridged into 9 themes by combining six themes into other similar themes and by deleting one theme. The final 9 themes were: Away from home: homesickness, school is challenging and stressful, it is not cold it is freezing, lack of physical activity, social norms vs. peer pressure, dietary patterns and available options, away from home and arriving to new home, time is money and day to day expenses. The selected themes were relevant to the study purpose and student responses were very descriptive in response to the opening statement of the interview.

\section{Theme 1: Away from home: homesickness}

The lifestyle changes among the interviewed subjects started with cultural shock after arriving from Saudi Arabia to the United States. They have had experienced homesickness at the beginning as they have left their 
families and started to live independently away from the their own society. One interviewee has two children, one is in the United States and one is back home with her mother.

"I have one child here and one there".

"I had a daughter here 8 months ago and she is with my mother back in Saudi, yeah when I first left her there I used to have a major homesickness".

However, they overcame homesickness by calling their families back home on daily basis, they have used video calls as well.

\section{Theme 2: School is challenging and stressful}

As they all received their undergraduate degrees from different schools than the current one, they considered the curriculum of the highly ranked school they are attending currently is challenging. One interviewee expressed that by saying:

"When I started my program everything was completely different than what I have studied back home, since my university is a research based university so it needs a lot of readings, so I need to read 2 to 3 papers weekly and to do some homework and to do some projects and programming. I spent like two months depressed and shocked because of the new lifestyle and educational system so this depressed me more and more and the problem with me when I am depressed I start gaining weight. This is my way to expelling the depression"

This educational differences put an extra pressure on them to over study to bridge the gap between their undergraduate and graduate studies. Moreover, two interviewees enrolled in undergraduate classes to fill the gaps in their knowledge or to fulfill some prerequisites.

"I am an international students so I have a lot of obstacles, one of them is English, so my English is not like the native American because it is their language, so sometimes I have difficulty in understanding the contents like them. The second obstacle maybe is the education here is better than other countries like the middle east, so you have to work twice as the people here who graduated from the United States maybe this is the reason you are busy all the time and you have to work hard all the time"

They were faced with a demanding curriculum where they are expected not to only read and work on projects, but to do more of literature reading and critiquing on weekly basis, to submit weekly assignments on top of the final projects and software programming each semester. An interviewee 
summarized her experience in one sentence:

"I am a full time student and it is very demanding"

This stressful situation put the students in a position where they devoted their entire time to their studies and ignored other recreational activities like exercise for instance. One subject explained it by stating that:

"I never think of going to the gym, the only thing I was thinking about is how to study, how to focus more every second and I think it is a psychological thing. Sometimes I spend 3 to 4 hours during the day without doing anything nor studying, just sitting, maybe watching TV or browsing Facebook or twitter or any social network, the thing is that you are thinking about your study, you are thinking about your work, how to complete your work, how to complete your research, how to complete your project"

\section{Theme 3: It is not cold, it is freezing}

The subjects found it hard for them and their families to get used to the very cold weather when they first arrived to New England. It was frustrating not being able to be outdoors with friends and family and staying indoors instead seeking warmth. They stated that:

"When I first came here was like -20 C in the winter so it was very hard for my family and me to get used to the weather"

"The northeast is the cold, so I live in a city back home which has a cold weather but of course not like here, it makes me always lazy, I like more to be warm during the study, if I want to study, if I want to focus more I need to stay in my room and to turn on the heater and stay warm"

\section{Theme 4: Lack of physical activity}

Due to the stressful studies, they have limited their physical activities and exercise and started to gain weight as they have expressed in their own words:

" I am here for about two years, I gained about 35 to 40 pounds, which is too much, too much, because I have nothing to do as I told you like I am in a tough school and they need a good work and they have deadlines and they want you to work hard”

"When I came here I found that I have sleep apnea and I gained $20 \mathrm{~kg}$ because I did not do any exercise as I was always studying and 24/7 on the computer which actually made my sleep apnea worse and I had trouble sleeping" 


\section{Theme 5: Social norms vs. peer pressure}

The social norms in the United States impacted that as well, as being overweight or obese is acceptable compared to the situation back in Saudi Arabia, where they used to exercise and watch their weight due to peer pressure on top of social norms. Here how one interviewee expressed it:

"People in the middle east are thinner and you feel like a strange person among them or a different person among them when you are over weight or obese, but when I came here to the United States it seems no body cares about you and no body asks why you are gaining weight".

He also mentioned the amount of weight he gained during a short period of time away from home:

"For less than seven months, seven to eight months, I gained about 25 to 30 pounds and I didn't realize that I gained weight as no body noticed my weight gain"

The same interviewee elaborated his experience with the social networks and their effects on his own lifestyle:

"I think lifestyle is affected by the social network, so if you have friends in a small social network and they observe something bad or good in you, they will tell you about it”.

He also stated that:

"When I was back home, we were 5 to 6 friend who were always together, going daily to the gym so you feel that you live in a healthy environment and you feel bad when you gain like 2 to 3 pounds, the social network really helps".

\section{Theme 6: Dietary patterns and available options}

All subjects were Muslims, despite the fact that they have some food restrictions due to their religion as they should stick to Halal food, the did not see it a problem when they select their dietary options, as their problem was with the type of food, as they don't cook during the weekdays and eat fast food instead, with all its unhealthy components which they all are aware of. One of the subjects said that everything he used to eat back in his country is not available in the town he is living in currently in the United States, so when he goes back home for a vacation, he brings back with him a stock of food items needed to cook his traditional food and recipes. Another one stated that "bad/junk" food is available as well in his country, but in the same time he has the option to have a healthy food, compared to his situation here where he is mostly eating fast food like pizza, burgers and french fries. Due to that, he gained 25-30 lb in 7 months. He is also selective in his meals due to religious restrictions of food items like pork and alcohol. He said:

"The food here is very bad compared to my country". 
In two years, he gained 35-40 lbs, his reason was:

"I have nothing to do as I told you like I am in a tough school and they need a good work and they have deadlines and they want you to work hard and you have to do twice work".

Another subject said that:

"I don't cook as I used to, I cook usually in the weekends and mostly we eat out, we did not use to eat much outside before coming here".

She also explained the reason for her food selections by saying:

"I try to avoid fatty food, but here it is difficult, and most of my diet is fast food, here it has been difficult, probably hard to avoid this as there is no a lot of places".

\section{Theme 7: Away from home and arriving to new home}

The living conditions were expressed as a lifestyle change, as they started to hold more responsibilities by taking care of their new places and to manage their expenses throughout the month. One subject started to have problems with his neighbor in the condo, as his neighbor downstairs get annoyed every time he or his family member moves around. He states that:

"This was the most annoying experience since I came to here, he used to call the police all the times and he nocks from downstairs and freaked my child out".

Another subject said that she did not feel that things have been changed in her life:

"Nothing changed actually as I live with my husband and child, I don't feel there is a lot of change".

However, she said that responsibilities at home have been increased "It is just me, I used to have help in my country, I don't have it here, now I clean by my self, I do the laundry and wash the dishes and everything".

Another subject expressed the same:

"I feel like when I moved to the new apartment and got married and took the responsibility of my own apartment and my own things I stayed close with everything here like the rent payment. As a husband I also take hospital appointments for my wife".

One subject complained about winter storms, due to frequent storms, he faced the problem of power outages, so he struggled by visiting to the community center and get water and supplies.

\section{Theme 8: Time is money}

The culture in the United States is different than their country as it has 
a faster pace and people are more organized and accurately on time. This has an advantage as the subjects focus more on their work and became more organized. One stated that:

"I am always in a rush I don't have time, I can make up one day for my family and the rest for my study, this was a major change in my lifestyle, because when I was In my country I can spend like 3 days a week with my family and when I come from work I can go out and do grocery shopping. Here, if you want to go and do grocery shopping it takes like 4 hours and I have to plan for it for the week, and if I want to buy something in the middle of the week it is a little bit hard because the nearest store is about 15 minutes away from my place".

He added:

"We started to changed my lifestyle and my child's lifestyle to make him go to bed to sleep at 9 o'clock".

Another subject said that being away from home and family has an advantage as she focuses more on her work:

"I feel that I have practical here, since I am away from my parents so I am more focused on my career, this is in general".

One subject expressed his reaction to this by stating

"I think the lifestyle in the United States is organized in case of time and time management, I think like when you live in the United States your daily schedule will be changed and become organized".

On the other hand, he feels that his lifestyle back home was better than here in the United States:

"My lifestyle was better than here in the Unites States in the case of health, in case of exercises in case of my time management. Actually, when I was back home all the thing I was thinking about was my health and how to go to the gym and how to lose weight".

\section{Theme 9: Day to day expenses}

It took them time till they managed their monthly expenses, it took one subject long time to manage his spending as he was afraid of running out of money before the next pay check. This is what he said:

"I took time till I managed my salary throughout the month as it differs here than home, it took me long time to manage that. I afraid that by the end of the month I run out of money."

He also added:

"The gas prices are much more expensive than back home." 


\section{The fundamental structure and discussion}

An exhaustive description was created from the themes and the formulated meaning was adjusted and validated by the interviewees, then the fundamental structure was developed accordingly. The fundamental structure refers to 'the essence of the experiential phenomenon as it is revealed by explication'. The discussion will follow the fundamental structure as instructed by the method used (Colaizzi, 1978).

\section{The fundamental structure of the phenomenon:}

The lifestyle changes among the interviewed subjects started with cultural shock after arriving from Saudi Arabia to the United States. They have had experienced homesickness at the beginning as they have left their families and started to live independently away from the their own society. However, they overcame homesickness by calling their families back home on daily basis, they have used video calls as well. As they all received their undergraduate degrees from different schools than the current one, they considered the curriculum of the highly ranked school they are attending currently is challenging. The educational differences put extra pressure on them to over study to bridge the gap between their undergraduate and graduate studies. Moreover, two interviewees enrolled in undergraduate classes to fill the gaps in their knowledge or to fulfill some prerequisites. They were faced with a demanding curriculum where they are expected not to only read and work on projects, but to do more of literature reading and critiquing on weekly basis, to submit weekly assignments on top of the final projects and software programming each semester. This stressful situation put the students in a position where they devoted their entire time to their studies and ignored other recreational activities like exercise for instance. The subjects found it hard for them and their families to get used to the very cold weather when they first arrived to New England. It was frustrating not being able to be outdoors with friends and family and staying indoors instead seeking warmth. Due to the stressful studies, they have limited their physical activities and exercise and started to gain weight. The social norms in the United States impacted that as well, as being overweight or obese is acceptable compared to the situation back in Saudi Arabia, where they used to exercise and watch their weight due to peer pressure on top of social norms. Despite the fact that they have some food restrictions due to their religion, the subjects do not see it a problem when they select their dietary options, as their problem was with the type of food, as they don't cook during the weekdays and eat fast food instead, with all its unhealthy components they all are aware of. The living conditions were expressed as a lifestyle change, as they started to hold more responsibilities by taking care of their new places and to manage their expenses throughout the month. The 
culture in the United States is different than their country as it has a faster pace and people are more organized and accurately on time. However, this has an advantage as the subjects focus more on their work and became more organized.

\section{Discussion}

The nine themes have explored how changes in the culture and environment would affect the behavior and lifestyle of the international students living in the United States. Themes 1, 2 and 3 identified and explored how being away from home and family and moving to a new country where the environment and the weather is extremely different would affect the way students started to live. Moreover, the stress of the challenging curriculum was another burden that made them staying mostly indoors and isolating themselves from the society. These findings are similar to what was reported by Al-Dabal, et al. (2010). This social impact was further explored in themes 4 and 5, where being stressed out by the hard study loads and being away from their social networks facilitated physical inactivity and lack of exercise which ended up in gaining weight in a considerably short periods of time. Weight gain was not only due to limited physical activity and lack of exercise, it was also because of their unhealthy dietary patterns and food selection as was identified and expressed in them 6, where the subjects eat more of unhealthy fast food instead of cooking and preparing their own meals. These findings are similar to those reported by Finlayson, et al. (2012) and by Alzayani and Hamadeh (2011). Themes 7 and 8 explored how living independently away from home affected their way of life as they became more organized and focused on their work, which is similar to what Lee (2004) has reported. They also started to hold more responsibilities, taking care of their own families, cleaning the house and managing their expenses, which was not an easy task as clearly explored in theme 9. These themes would possibly be generalized to students coming from the Middle East as they share the same cultural background. However, it is not necessarily to conform to those coming from other regions in the world or even domestically in the United States.

\section{Conclusion}

From this study, we can see that despite changes in culture and environment, students' behavior and lifestyle have been affected in different ways, some were positively affected like being more time efficient and focusing more on work, and some were negatively affected like being under stress, eating unhealthy diet and physical inactivity. Students were aware of the changes in their lifestyle and were always trying to modify them. Students counseling services should be available in the schools for the 
international students to help them and their families to handle the changes they face before starting and during their studies in the Unites States.

\section{References:}

Al-Dabal, B. K., Koura, M. R., Rasheed, P., Al-Sowielem, L., \& Makki, S. M. (2010). A comparative study of perceived stress among female medical and non-medical university students in dammam, saudi arabia. Sultan Qaboos University Medical Journal, 10(2), 231-240.

Alzayani, S. H., \& Hamadeh, R. R. (2011). Use of electronic communication and entertainment devices: A survey of arabian gulf university medical students. Bahrain Medical Bulletin, 33(3)

Colaizzi, P. (1978). Psychological research as the phenomenologist's view it. In R. Valle, \& M. King (Eds.), Existential-phenomenological alternatives for psychology (pp. 48-71). New York: Oxford University Press.

Deluca, E. K. (2005). Crossing cultures: The lived experience of jordanian graduate students in nursing: A qualitative study. International Journal of Nursing Studies, 42(6), 657-663. doi: 10.1016/j.ijnurstu.2004.09.017

Edward, K. L., \& Welch, T. (2011). The extension of Colaizzi's method of phenomenological enquiry. Contemporary Nurse, 39(2), 163-171. doi: 10.5172/conu.2011.163

Finlayson, G., Cecil, J., Higgs, S., Hill, A., \& Hetherington, M. (2012). Susceptibility to weight gain. eating behaviour traits and physical activity as predictors of weight gain during the first year of university. Appetite, 58(3), 1091-1098. doi: 10.1016/j.appet.2012.03.003

Kohl, H. W.,3rd, Craig, C. L., Lambert, E. V., Inoue, S., Alkandari, J. R., Leetongin, G., . . . Lancet Physical Activity Series Working Group. (2012). The pandemic of physical inactivity: Global action for public health. Lancet, 380(9838), 294-305. doi: 10.1016/S0140-6736(12)60898-8

Lee, N. J. (2004). The impact of international experience on student nurses' personal and professional development. International Nursing Review, 51(2), 113-122. doi: 10.1111/j.1466-7657.2003.00200.x

Supranowicz, P. (2011). Evaluation of usefulness of summarised and weighted scale of negative life events to examine the determinants of adolescent health. [Porownanie przydatnosci sumarycznej i wazonej skali negatywnych zdarzen zyciowych do badania uwarunkowan zdrowia mlodziezy] Roczniki Panstwowego Zakladu Higieny, 62(3), 307-314. 